Article

\title{
Adaptive Gains Control Scheme for PMSG-Based Wind Power Plant to Provide Voltage Regulation Service
}

\author{
Jianfeng Dai ${ }^{1}$, Yi Tang ${ }^{1, *}$ and Jun $\mathrm{Yi}^{2}$ \\ 1 School of Electrical Engineering, Southeast University, Nanjing 210096, China; daijianfeng@seu.edu.cn \\ 2 China Electric Power Research Institute, Beijing 100192, China; 220172668@seu.edu.cn \\ * Correspondence: tangyi@seu.edu.cn; Tel.: +86-25-8379-0617
}

Received: 10 January 2019; Accepted: 20 February 2019; Published: 24 February 2019

\begin{abstract}
High-penetration wind power will count towards a significant portion of future power grid. This significant role requires wind turbine generators (WTGs) to contribute to voltage and reactive power support. The maximum reactive power capacity (MRPC) of a WTG depends on its current input wind speed, so that the reactive power regulating ability of the WTG itself and adjacent WTGs are not necessarily identical due to the variable wind speed and the wake effect. This paper proposes an adaptive gains control scheme (AGCS) for a permanent magnet synchronous generator (PMSG)-based wind power plant (WPP) to provide a voltage regulation service that can enhance the voltage-support capability under load disturbance and various wind conditions. The droop gains of the voltage controller for PMSGs are spatially and temporally dependent variables and adjusted adaptively depending on the MRPC which are a function of the current variable wind speed. Thus, WTGs with lower input wind speed can provide greater reactive power capability. The proposed AGCS is demonstrated by using a PSCAD/EMTDC simulator. It can be concluded that, compared with the conventional fixed-gains control scheme (FGCS), the proposed method can effectively improve the voltage-support capacity while ensuring stable operation of all PMSGs in WPP, especially under high wind speed conditions.
\end{abstract}

Keywords: permanent magnet synchronous generator (PMSG); droop control; adaptive-gain; voltage support; maximum reactive power capacity (MRPC)

\section{Introduction}

Wind power is increasingly integrated into the modern power system and will be a major source of electrical power in the near future [1-3]. However, the unpredictable nature of wind causes random fluctuations in wind power, which increases the instability risk of wind power grid-connected systems. The voltage of a power system should always be within the allowable range to ensure safe and reliable operation.

Recently, variable-speed wind turbine generators (WTGs) such as the permanent magnet synchronous generator (PMSG) and doubly fed induction generator (DFIG) have become more popular because of the maximum power tracking operation and flexible additional controls by using converters [4], in which a PMSG is widely used in the wind power system for its advantages of lower maintenance cost, high reliability and flexible control. However, the fixed reactive power control of the wind turbine (WT) converter causes them not to respond to the system voltage variation. As a result, the voltage of the system will drop significantly without other reactive power compensation devices when an external disturbance occurs, especially in a power system with a high wind power penetration level. In order to minimize this issue and improve the ability of the power grid to accommodate more 
wind power, power system operators have imposed strict grid codes for larger wind-power plants (WPPs). One of the mandatory grid codes is that a WPP must be equipped with functions of reactive power and voltage control, including the voltage ride through under fault conditions and reactive power provision under steady states [5-7].

A number of research works on the voltage-supporting schemes of WPPs have been reported. One of the research hotspots is the automatic voltage control (AVC) system for wind power plants, which is used to control the reactive power of reactive power compensation devices in wind power plants according to the dispatching instructions of the power grid [8-10], so as to meet the reactive power and voltage requirements of the grid connection point. In [8], the authors designed a hierarchical automatic voltage control system to support wind power integration, in which the voltage and reactive power distribution inside the wind power plant are optimized and three different control modes are implemented considering all the terminal voltages of WTGs as well as the dynamic var reserves. In [9], the authors proposed a coordination strategy between an automatic voltage regulator (AVR) and power system stabilizer (PSS) of synchronous generators, and fuzzy logic is used to solve the problem. In [10], an automatic voltage control system for power systems with limited continuous voltage control capability is presented. Voltage uncertainty caused by wind power forecasting errors is considered in the optimal voltage/var dispatch by including a security margin in the voltage magnitude constraints.

However, the analysis of reactive power compensation and voltage stability in WPP is mostly based on static var compensator (SVC), static var generator (SVG) or on-load tap changer and other local compensation devices for the AVC system mentioned above, and the WTG's own reactive power capability has not been considered. Actually, the WTG has the ability to generate some reactive power both from the machine-side converter (MSC) and from the grid-side converter (GSC). Therefore, the WTG can be regarded as a continuous reactive power source. The ability of reactive power adjusting under two conditions (with and without crowbar) are analyzed in [11]. In [12], the authors present the supervisory reactive power control scheme in order to regulate the voltage at a remote location. In [13], a dynamic reactive power allocation scheme based on autonomous voltage security regions to accommodate more wind power is proposed in large-scale centralized wind power integration areas. Benders decomposition is used to solve the allocation scheme.

However, in the above literature, the fix droop gain of the voltage controller for each WTG is used. As a result, difficulty arises in finding a proper gain of a WTG for various wind conditions. A larger gain has better voltage support performance, but may exceed the maximum reactive power capacity (MRPC) resulting in increasing wear and tear to the converters. Conversely, a small gain ensures stable operation, but the performance of voltage support is limited. More importantly, if the fixed-gains control scheme (FGCS) is applied to a WPP consisting of multiple WTGs, it increases the difficulty of finding the same suitable gain for all WTGs, which have different levels of MRPC due to fluctuating wind speed and wake effects [14-17]. Thus, WTGs in a WPP operate at different maximum output power points, and each WTG has different MRPC depending on the current input wind speed. This means that a larger droop gain is suitable for downstream WTGs that have higher MRPC level, but it is unsuitable for upstream WTGs that have a lower MRPC level, and vice versa.

This paper proposes an adaptive droop gains control scheme for PMSG-based WPP that discriminates the PMSG's contribution to provide voltage regulation service. The proposed control scheme aims to make full use of the MRPC retained in a WPP to improve the voltage support performance while ensuring stable operation of all PMSGs, especially under the high wind speed conditions. To achieve this, the adaptive droop gain of each PMSG is set to be proportional to its current MRPC that is a function of current wind speed and is spatially and temporally dependent due to the wake effect, so that a PMSG with a large MRPC makes a greater contribution to voltage regulation by setting a larger droop gain, and a PMSG with a small MRPC ensures stable operation during voltage control by setting a smaller droop gain. The effect of the proposed adaptive gains control scheme (AGCS) is compared with conventional FGCS and no voltage control loop scheme. The 
validity of this proposed strategy is extensively demonstrated under various wind conditions using a PSCAD/EMTDC simulator.

\section{Proposed Voltage Support Scheme Based on Adaptive Gains}

This section briefly describes the PMSG model used in this paper and the FGCS for reactive power and voltage control, and then the overall features of the proposed AGCS are described in detail.

\subsection{Permanent Magnet Synchronous Generator (PMSG) Model}

A typical configuration of the PMSG model is shown in Figure 1, which consists of wind turbines, PMSGs, full-power converters and control systems. The PMSG is operated with a combination of maximum power point tracking (MPPT) strategy and pitch control. Table 1 gives the parameters of a PMSG studied in this paper [18].

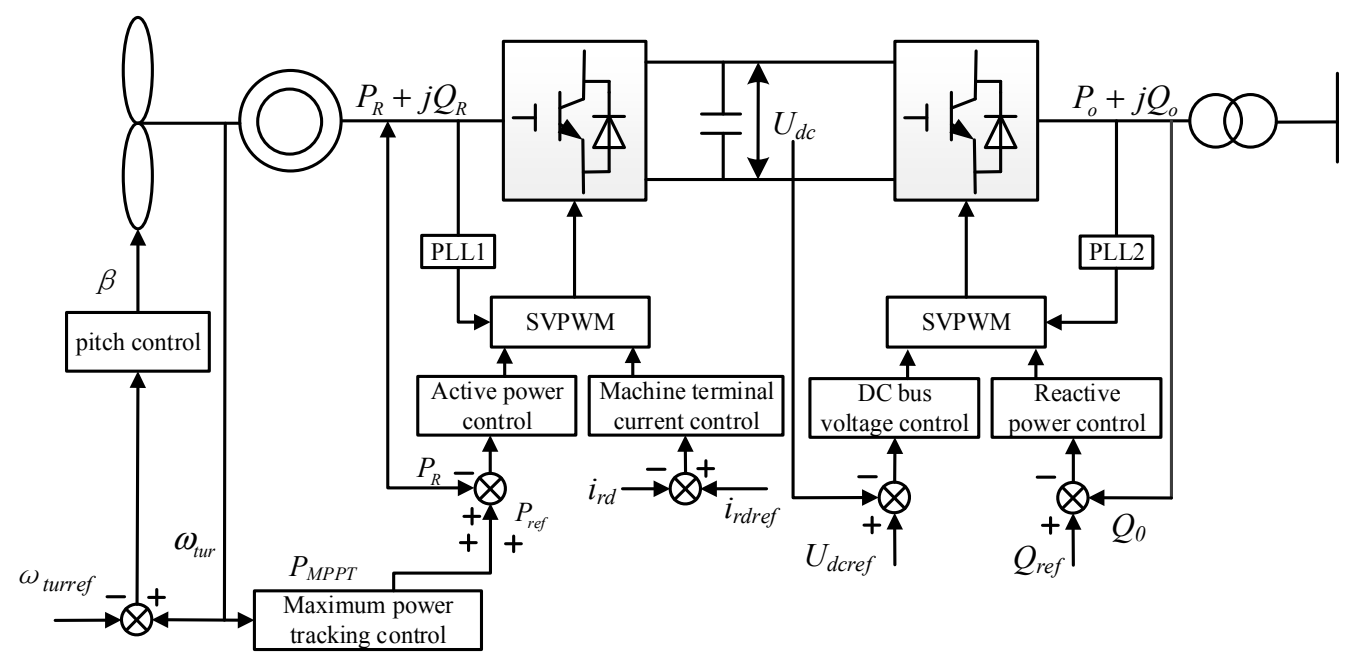

Figure 1. A typical configuration of the permanent magnet synchronous generator (PMSG) model.

Table 1. Parameters of a PMSG used in this paper.

\begin{tabular}{ccc}
\hline Parameters & Units & Values \\
\hline Nominal Apparent Power & MVA & 2 \\
Nominal Stator Voltage & $\mathrm{kV}$ & 0.69 \\
Stator Resistance & p.u. & 0.0108 \\
Stator Leakage Reactance & p.u. & 0.102 \\
Rotor Resistance & p.u. & 0.01 \\
Rotor Leakage Reactance & p.u. & 0.11 \\
Inertia Time Constant & s & 3 \\
\hline
\end{tabular}

A PMSG controller measures signals such as voltage, current and rotor speed to provide signals for the control system that consist of the MSC and the GSC. A typical GSC control scheme is shown in Figure 2. The active and reactive power of a PMSG can be decoupled independently, which is achieved by controlling the active components $i_{\mathrm{d}}$ and reactive components $i_{\mathrm{q}}$ of the grid-connected current.

The mechanical power equation of WTG extracted from the wind is expressed as:

$$
P_{t}=\frac{1}{2} \rho \pi r^{2} v^{3} C_{p}(\lambda, \beta)
$$

where $\rho, r, v, C_{p}, \lambda$ and $\beta$ are the air density, blade length, wind speed, coefficient of performance of the wind turbine $(\mathrm{WT})$, tip-speed ratio and blade pitch angle respectively. 


$$
C_{p}=(0.44-0.0167 \beta) \sin \left[\frac{\pi}{2} \frac{(\lambda+1)}{7.8-0.15 \beta}\right]
$$

The expression for $\lambda$ is shown as:

$$
\lambda=\frac{\omega_{t u r} r}{v}=\frac{2 \pi r n_{t u r}}{v}
$$

where $\omega_{t u r}$ is the angular velocity of the WT; $n_{t u r}$ is the pole number of the WT.

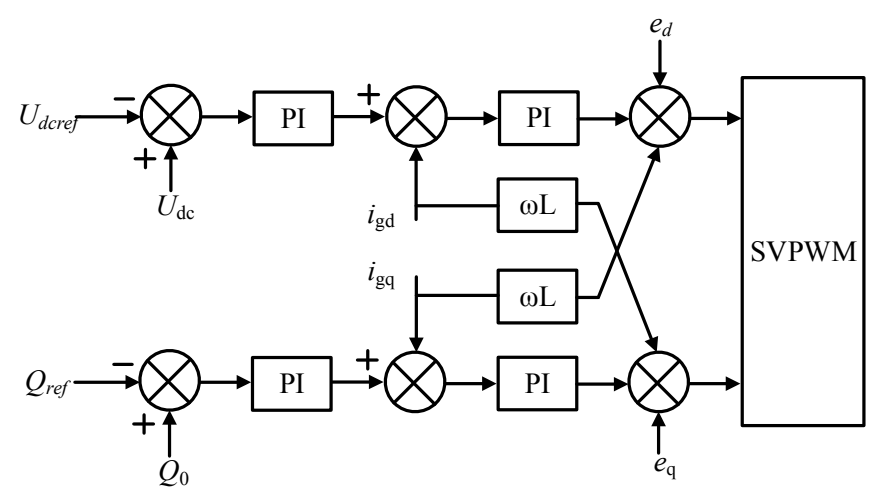

Figure 2. Control strategy of grid-side converter.

At a given wind speed, maximum power can be extracted from a WT for a specific rotor speed and pitch angle. In general, when the WT output is less than the rated power, the pitch angle is maintained at a fixed value. At this point, $C_{p}$ is a function of $\lambda$. By adjusting the angular speed of the wind turbine, the maximum $C_{p}$ is achieved with maximum power tracking control. So $\lambda$ and $C_{\mathrm{p}}$ can take an optimal value of $\lambda_{\text {opt }}$ and $C_{\text {pmax }}$, and the output power of each WT can be expressed as:

$$
P_{t}=\frac{1}{2} \rho \pi r^{2} v^{3} C_{p \max }(\lambda, \beta)=k_{o p t} v^{3}
$$

where $k_{\text {opt }}$ is the equivalent coefficient to obtain the maximum wind energy.

The reactive power capacity of WTG is determined by the active power from the grid-side of the generator and the apparent power of the converter. Assuming that the active power loss is ignored, the reactive power capacity of the WTG can be expressed as:

$$
Q_{W}= \pm \sqrt{S_{W}^{2}-P_{t}^{2}}
$$

where $S_{W}$ is the apparent power of the WT converter.

It should be emphasized that in extreme cases, when the reactive power shortage is large and all WTGs are running at full power, the upper limit of the full power converter current is reached. Considering that the reactive power adjustment capability of the WTG is closely related to the active power generated, the deloading operation of WTG is considered to increase the reactive power margin. The corresponding deloading adjustment methods include over-speed operation and pitch angle control [19]. Then, the reactive power capacity of WTG can be expressed as

$$
Q_{W}= \pm \sqrt{S_{W}^{2}-\left[(1-d) P_{t}\right]^{2}}= \pm \sqrt{S_{W}^{2}-(1-d)^{2} k_{o p t}^{2} v^{6}}
$$

where $d$ is the deloading level, and the range of value is usually $0-20 \%$.

According to Equation (6), Figure 3 illustrates the allowable range of active and reactive power output of a PMSG under different wind speeds. As can be seen from the figure, when the input wind speed of the WT is higher, the maximum allowable reactive power capacity is smaller, and the reactive power margin can be improved by sacrificing active power to the operation in the deloading operation 
state. Therefore, the reactive power regulation of the WT with low input wind speed is greater than that of the WT with high input wind speed. When the input wind speed of WT is greater than or equal to the rated wind speed, there is almost no reactive power regulation capacity if not sacrificing active power output.

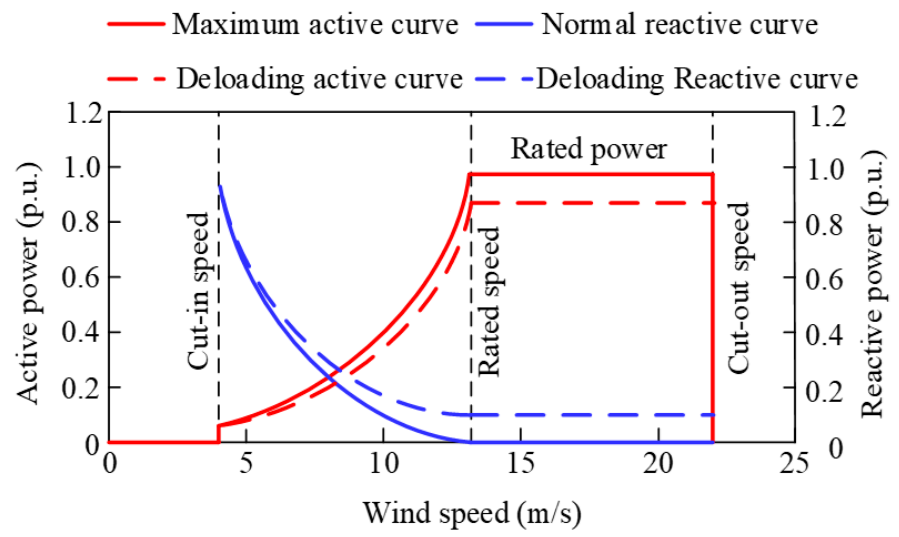

Figure 3. Active and reactive power range of the PMSG.

\subsection{Conventional Fixed-Gain Voltage Control Scheme}

This section briefly describes a conventional reactive power and voltage droop control schemes for permanent magnet synchronous generators (PMSGs) [20,21], which uses an additional fixed-gain droop loop, as shown in Figure 4. $Q_{r e f}$ consists of two terms: $Q_{0}$ and $\Delta Q$. In general, the value of $Q_{0}$ is zero.

$$
\Delta Q_{i}=-\frac{1}{R}\left(V_{\text {sys }}-V_{\text {nom }}\right)
$$

where $V_{\text {nom }}$ is the nominal voltage in p.u. at PCC in a WPP; $V_{\text {sys }}$ is the measured voltage in p.u. at PCC; and $1 / R$ is the droop loop gain.

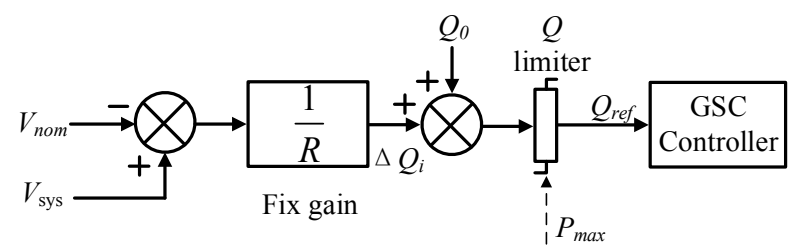

Figure 4. Conventional fixed-gain voltage control scheme of a PMSG.

Note that the droop gain $1 / R$ used in (7) is fixed. This means that if the value of $1 / R$ is too large, it is beneficial to improve the voltage regulation ability, but it will make the PMSG converters frequently exceed the limit of maximum reactive power, especially in the case of extremely narrow reactive power range of the PMSG, and this phenomenon is more likely to happen when the wind speed is high. Conversely, a small gain ensures stable operation of a PMSG, but it provides a limited contribution for voltage regulation. Therefore, inappropriate droop gain will lead to unsatisfactory results, and the conventional FGCS is unable to ensure both voltage regulation performance and stable operation of a PMSG at the same time. Moreover, it is worth noting that when the wind speed fluctuates frequently, the fixed gain scheme is more inappropriate.

\subsection{Wake Effect Model}

The Park wake model which is based on the Jensen wake model is used in this paper [22]. The model obtains the input wind speed of adjacent WTs by considering the wake effect. Assuming that the wake wind speed is linear expansion, and the wind speed of adjacent WTs is obtained by considering 
the cumulative effect of multiple shadows and the wind direction. The relationship between input wind speed of adjacent WTs can be expressed as:

$$
v_{i}=v_{0}\left[1-\sqrt{\sum_{\substack{j=1 \\ j \neq i}}^{n}\left\{2 a_{j}\left(\frac{D_{j}}{D_{j}+2 k x_{j i}}\right)^{2} \beta_{j i}\right\}^{2}}\right]
$$

where $v_{i}$ is the wind speed of the $i$-th wind turbine (WTi); $v_{0}$ is the wind speed; $a_{j}$ is the axial induction factor of the $j$-th wind turbine generator (WTGj); $D_{i}$ is the diameter of swept area of the $i$-th wind turbine generator (WTGi); $k$ is the wake-decaying constant; $x_{j i}$ is the radial distance between WTGj and WTG $i ; \beta_{j i}$ is the ratio between the overlapping area and swept area of WTG $i$; and $n$ is the total number of the WTs.

\subsection{Adaptive Gains Voltage Support for a PMSG-Based Wind-Power Plants (WPP)}

As mentioned in the previous section, PMSGs within a WPP have different levels of reactive power capability due to the fluctuation of wind speed and the wake effect. The proposed adaptive gains voltage support scheme aims to improve the voltage nadir while ensuring stable operation of a PMSG-based WPP. The configuration of the proposed adaptive gain scheme of a PMSG is shown in Figure 5. In the proposed scheme, the output of the droop control loop can be defined as:

$$
\Delta Q_{i}=-A G_{i}\left(v_{i}\right)\left(V_{s y s}-V_{\text {nom }}\right)
$$

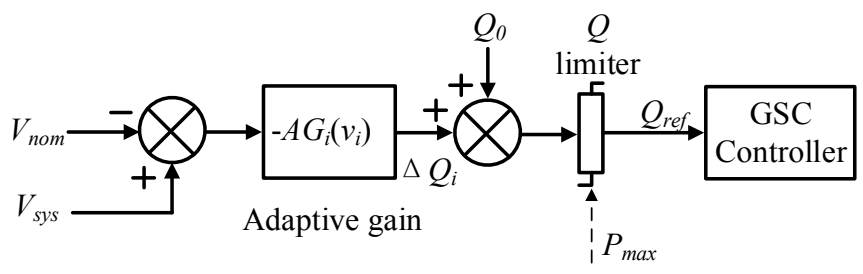

Figure 5. Proposed adaptive-gain voltage control scheme of a PMSG.

To achieve better voltage regulation performance and ensure stable operation at the same time, the control gain of the voltage deviation loop of PMSGi, $A G_{i}\left(v_{i}\right)$, is set to be proportional to the current MRPC of PMSG $i, Q_{i}$, which can be expressed as:

$$
A G_{i}\left(v_{i}\right) \propto Q_{i}=\sqrt{S_{W}^{2}-(1-d)^{2} k_{o p t}^{2} v_{i}^{6}}
$$

In this paper, in order to obtain the proportionality constant of (10), (10) can be rewritten in the following form:

$$
A G_{i}\left(v_{i}\right)=\frac{1}{R_{0}} \frac{Q_{i}}{Q_{\max }}
$$

where $Q_{\max }$ is the maximum value of $Q_{i}$, and can be obtained by inserting the cut-in wind speed in to (6). $1 / R_{0}$ is the maximum droop loop gain. The value of $R_{0}$ can be determined as different values depending on the design purposes.

Rearranging (11) gives:

$$
A G_{i}\left(v_{i}\right)=\frac{1}{R_{0}} \frac{\sqrt{S_{W}^{2}-(1-d)^{2} k_{o p t}^{2} v_{i}^{6}}}{\sqrt{S_{W}^{2}-k_{o p t}^{2} v_{c u t-i n}^{6}}}
$$


where $v_{\text {cut-in }}$ is the cut-in wind speed.

Thus, the output of the adaptive gain droop control loop can be expressed as:

$$
\Delta Q_{i}=\frac{1}{R_{0}} \frac{\sqrt{S_{W}^{2}-(1-d)^{2} k_{o p t}^{2} v_{i}^{6}}}{\sqrt{S_{W}^{2}-k_{o p t}^{2} v_{c u t-i n}^{6}}}\left(V_{s y s}-V_{n o m}\right)
$$

In (13), $v_{i}$ and $A G_{i}\left(v_{i}\right)$ are spatially and temporally dependent variables. First, the reason for the spatial dependency is that each PMSG has a spatially different level of current MRPC due to the wake effect between the adjacent PMSGs. In order to obtain better voltage support performance, a larger gain is set for a downstream PMSG converter that has a more MRPC (less active power generation), whereas a small gain is set for an upstream PMSG converter that has a less MRPC (more active power generation). In addition, the droop gain is set to zero when the wind speed $v_{i}$ is greater than or equal to the rated wind speed. This helps PMSG-based WPP to provide more voltage support and ensure stable operation. Second, the reason for the temporal dependency is that the input wind speed of WT is time-varying. As a result, the MRPC of each PMSG is also time-varying, and droop gain is adjusted adaptively with the change of input wind speed. Therefore, the proposed AGCS can improve the voltage support and ensure the stable operation when set to be proportional to the spatially and temporally different MRPC. It provides effective contribution to voltage regulation by increasing the droop gains depending on the increasing MRPC and thus prevents the PMSGs from reaching the maximum reactive power operation limit frequently by reducing the droop gains depending on the decreasing MRPC, which helps avoid the wear and tear of the PMSG converters.

\section{Model System}

Figure 6 shows a test system used in this paper to investigate the performance of the voltage control schemes. The test system consists of a synchronous generators (SG) of $100 \mathrm{MW}$ [23], a static load of $30 \mathrm{MW}$ and 1 MVAR, and 12 2-MW PMSG-based WPP. In order to differentiate each PMSG, each column and each row of the PMSG are numbered from WT1 to WT12 separately, as shown in Figure 6.

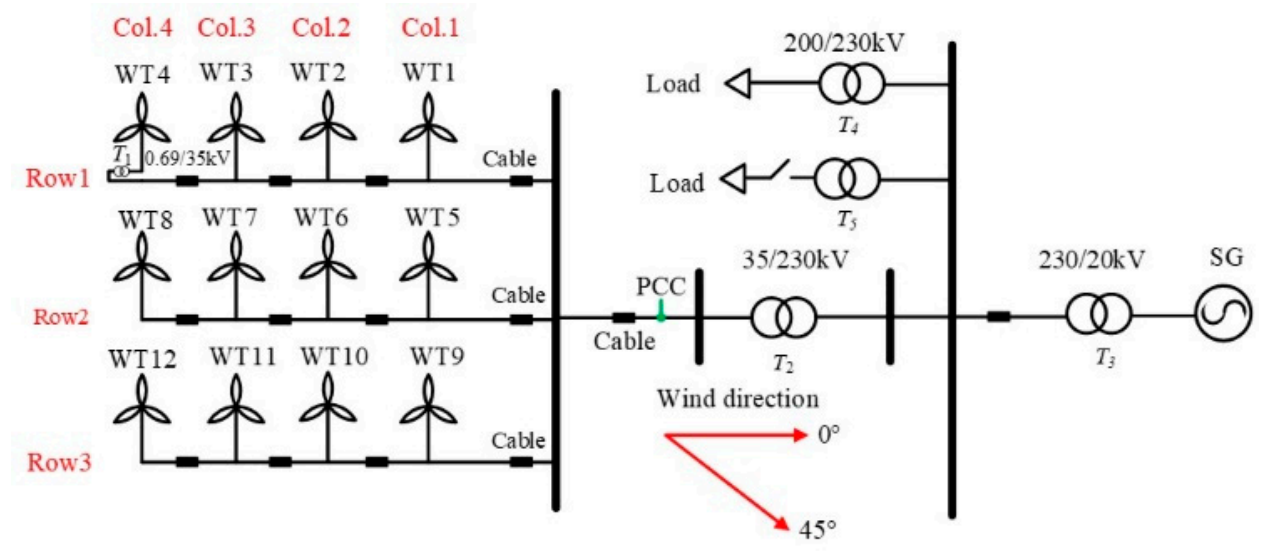

Figure 6. Test system used in this paper.

\subsection{Synchronous Generators}

The SG is a steam turbine generator with a rated capacity of $100 \mathrm{MVA}$, and it is equipped with excitation adjustment function. Figure 7 and Table 2 show the IEEE alternator supplied rectifier excitation system (from [24]) and its coefficients, respectively. 


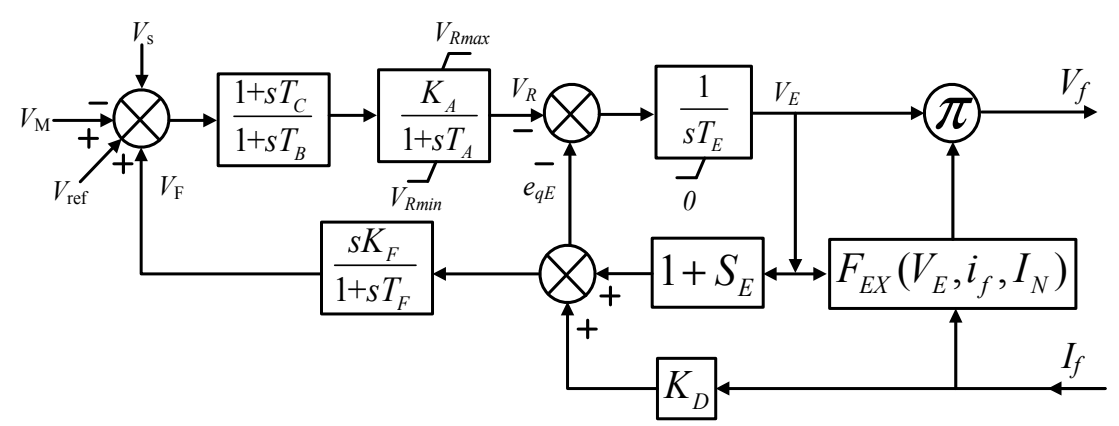

Figure 7. IEEE alternator supplied rectifier excitation system model.

Table 2. Coefficients of the IEEE alternator supplied rectifier excitation system model.

\begin{tabular}{ccccccccc}
\hline$T_{\mathbf{C}}$ & $\boldsymbol{T}_{\mathbf{B}}$ & $\boldsymbol{K}_{\mathrm{A}}$ & $\boldsymbol{T}_{\mathrm{A}}$ & $\boldsymbol{K}_{\mathrm{F}}$ & $\boldsymbol{T}_{\mathrm{F}}$ & $\boldsymbol{T}_{\mathrm{E}}$ & $S_{\mathrm{E}}$ & $\boldsymbol{K}_{\mathrm{D}}$ \\
\hline 0 & 0 & 400 & 0.02 & 0.03 & 1 & 0.8 & 4.18 & 0.38 \\
\hline
\end{tabular}

SVG, well-known as rapid dynamic reactive power compensation device, is not considered because this paper focuses on the reactive voltage control inside the WPP itself, and thus only the alternator supplied rectifier excitation system is considered. As a result, the voltage at the PCC is not fully recovered to the initial value after the disturbance event.

\subsection{PMSG-Based WPP}

The WPP consists of 12 2-MVA PMSGs. The PMSG is connected to the PCC through the $0.69 / 35-\mathrm{kV}$ transformer and connected to the grid (modelled by the large SG and the 230/20-kV step-up transformer) through the 35/230-kV transformer. The distance between the adjacent WTs is $1120 \mathrm{~m}$, and the distance between the $35 \mathrm{kV}$ bus and the SG is $20 \mathrm{~km}$. In PMSG-based WPP, it is assumed that the rated capacity of each WT in a WPP is the same, and the operating parameters, main circuit and control parameters of each WTG are also the same.

Similarly, as shown in Figure 3, the cut-in, rated, and cut-out wind speeds are 4, 13.2, and $22 \mathrm{~m} / \mathrm{s}$, respectively. Thus the output of PMSGs in WPP is not at the same level due to the wake effect between the adjacent PMSGs and the input wind speed of WT is time-varying. If the PMSG operates above the real active power, it will not have reactive power regulation capability. The voltage deviation at the PCC point is multiplied with adaptive gain $A G_{i}\left(v_{i}\right)$, and this result is added to the reactive power reference value.

In this paper, the PMSG controller measures the voltage at the PCC, and then the voltage RMS at the PCC point for voltage control is calculated.

\section{Case Studies}

Time-domain simulations are used to verify the proposed AGCS reported in the previous sections. The test system is modelled by using a PSCAD/EMTDC simulator [25]. According to the previous analysis, it can be seen that the performance of AGCS is closely related to wind conditions (i.e., wind direction and wind speed) and the disturbance of reactive power load. Thus, five cases have been implemented by varying the above conditions to compare the effectiveness of the proposed AGCS with respect to FGCS, in which $1 / R_{0}$ is set to the fixed value of 35 . In all cases, a static load of $12 \mathrm{MW}$ and 9 MVAR is added as a disturbance event at $2 \mathrm{~s}$; due to the voltage sags not being particularly large, the deloading level $\mathrm{d}$ of WTGs are set to 0 , namely $d=0$, and it should be emphasized that in the scenario of the large voltage drop caused by short circuit fault, when the reactive power margin of WPP is insufficient, the deloading operation of WTGs can be considered. 
In Case 1 and Case 2 the wind speed is assumed to be constant during the voltage control, and in Case 3 and Case 4 the wind speed is assumed to be increased at the instant of the event, from 13 to $14 \mathrm{~m} / \mathrm{s}$. In case 5, a real wind speed pattern is considered, that is, the wind speed is randomly fluctuating within the simulation time.

\subsection{Constant Wind Speed}

The voltage control capability of a WPP depends on the MRPC of the PMSG which is closely related to wind conditions (i.e., wind direction and wind speed). Thus this section describes the effects of high wind speed of $13 \mathrm{~m} / \mathrm{s}$ with $45^{\circ}$ and $0^{\circ}$ wind direction on the voltage control performance.

\subsubsection{Case 1: Wind Speed of $13 \mathrm{~m} / \mathrm{s}$, and Wind Direction of $45^{\circ}$}

Figure 8 shows the results when the constant wind speed is as high as $13 \mathrm{~m} / \mathrm{s}$ and wind direction is $45^{\circ}$. In this case, the wind speed of WT1, WT2, WT3, WT4, WT8, WT12 is $13 \mathrm{~m} / \mathrm{s}$, whereas due to the wake effect in (6), the wind speed of WT5, WT6, WT7, WT11 is $11.68 \mathrm{~m} / \mathrm{s}$, and the wind speed of WT9, WT10 is $10.97 \mathrm{~m} / \mathrm{s}$, as shown in Table 3. This means that the PMSGs in the fourth column and first row have largest active power and thus less MRPC, whereas other PMSGs have larger MRPC. The MRPC of all PMSGs obtained by using (6) and the droop gains obtained by using (12) are shown in Table 4, Figure 8 and Table 5, respectively. The wind speed, MRPC and droop gain are divided into three groups.

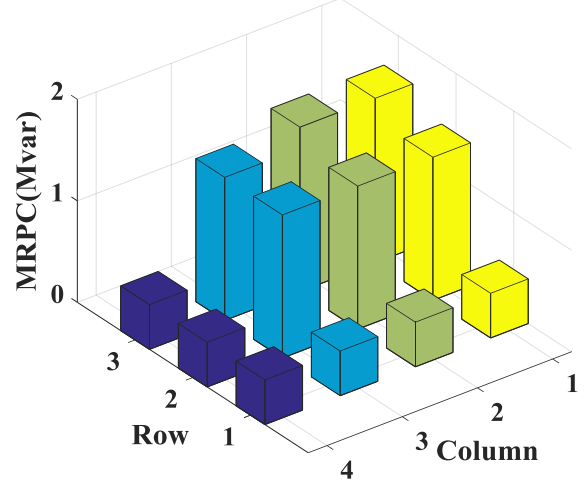

Figure 8. Maximum reactive power capacity (MRPC) of the all PMSGs in case 1.

Table 3. Wind speed of Case $1(\mathrm{~m} / \mathrm{s})$.

\begin{tabular}{cccc}
\hline Col.1 & Col.2 & Col.3 & Col.4 \\
\hline 13 & 13 & 13 & 13 \\
13 & 11.68 & 11.68 & 11.68 \\
13 & 11.68 & 10.97 & 10.97 \\
\hline
\end{tabular}

Table 4. MRPC of Case 1 (Mvar).

\begin{tabular}{cccc}
\hline Col.1 & Col.2 & Col.3 & Col.4 \\
\hline 0.44 & 0.44 & 0.44 & 0.44 \\
0.44 & 1.41 & 1.41 & 1.41 \\
0.44 & 1.41 & 1.62 & 1.62 \\
\hline
\end{tabular}

Table 5. Droop gain of Case 1.

\begin{tabular}{llll}
\hline Col.1 & Col.2 & Col.3 & Col.4 \\
\hline 16.04 & 16.04 & 16.04 & 16.04 \\
16.04 & 51.40 & 51.40 & 51.40 \\
16.04 & 51.40 & 59.07 & 59.07 \\
\hline
\end{tabular}


As shown in Figure 9a, the voltage nadir of three schemes (i.e., no voltage control, FGCS and AGCS) after the disturbance is 0.889 p.u., 0.949 p.u. and 0.957 p.u., respectively. Apparently, the steady state voltage of the proposed scheme is higher than that of the fixed scheme by 0.008 p.u. and higher than that of the "no voltage control scheme" by 0.068 p.u.. In the case of the same total reactive power demand, the proposed scheme shows a better performance on the voltage regulation than that of the FGCS.

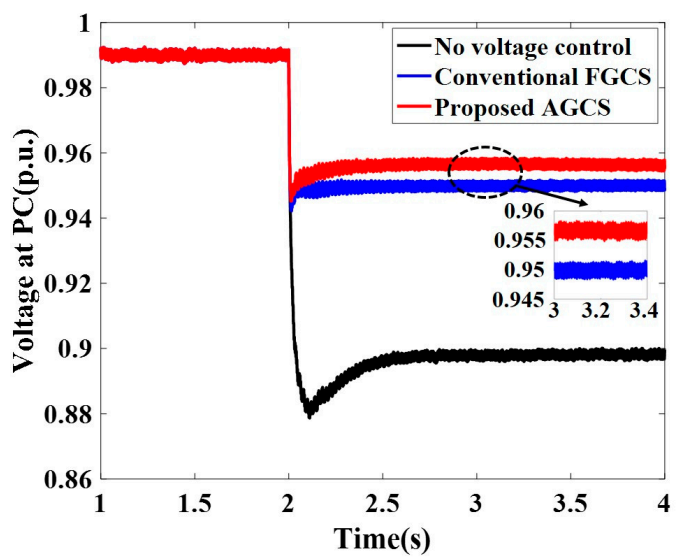

(a)

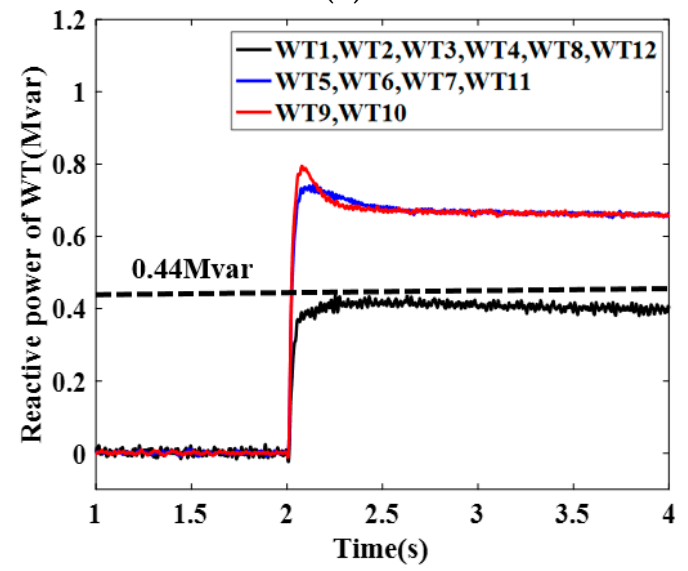

(b)

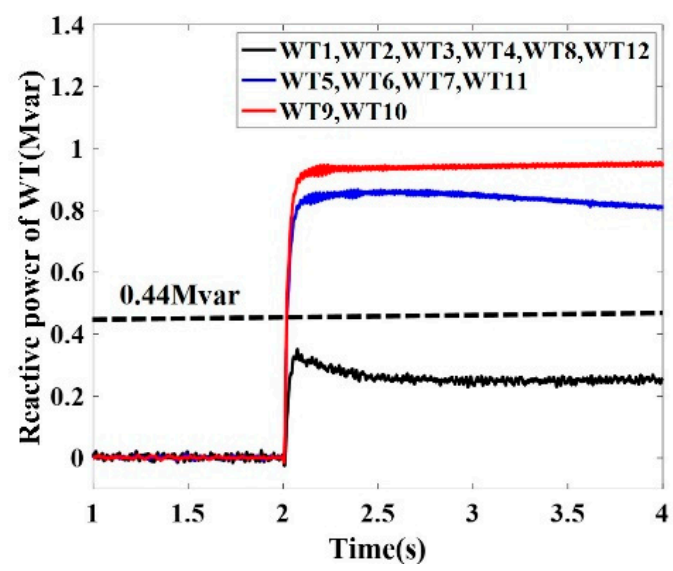

(c)

Figure 9. Results of Case 1. (a) Voltage at PCC, (b) reactive power of PMSGs under the conventional FGCS, (c) reactive power of PMSGs under the proposed AGCS. 
As shown in Figure 9b, for the FGCS, all PMSGs have the same reactive power generation within the MRPC range, whereas the reactive power generated by the fourth column and first row WTs (i.e., WT1, WT2, WT3, WT4, WT8, WT12) is constrained to 0.44 Mvar due to the maximum reactive power operating limits. The result is increasing wear and tear to the converters.

As shown in Figures 8 and $9 b, c$, the adaptive gain and thus the reactive power generation has a large value for the WTs that has smaller input wind speed, depending on the spatially different level of MRPC. The adaptive gains and the reactive power generation of WTs (i.e., WT1, WT2, WT3, WT4, WT8, WT12) are smaller than that of the FGCS, whereas the adaptive gains and the reactive power generation of other WTs are larger than that of the FGCS. This helps PMSGs to generate a certain amount of reactive power, while ensuring the stable operation of PMSGs without reactive power limits. In addition, it can be seen from Figure $9 \mathrm{c}$ that the upstream WTs have less reactive power generation than the downstream WTs at the wind directions, namely, reactive power generation of WTs (i.e., WT5, WT6, WT7, WT11) are larger than that of WTs (i.e., WT1, WT2, WT3, WT4, WT8, WT12) and are smaller than that of WTs (i.e., WT9, WT10).

\subsubsection{Case 2: Wind Speed of $13 \mathrm{~m} / \mathrm{s}$, and Wind Direction of $0^{\circ}$}

In this case, the wind direction is changed to $0^{\circ}$, and the MRPC of the downstream PMSGs is different from that in Case 1. The wind speed of each WT is shown in Table 6. The PMSGs in the fourth column have largest active power and less MRPC, whereas other PMSGs have larger MRPC. The MRPC of all PMSGs and the droop gains are shown in Table 7, Figure 10 and Table 8, respectively. The wind speed, MRPC and droop gain are divided into four groups, and each column is regarded as a group.

Table 6. Wind speed of case $2(\mathrm{~m} / \mathrm{s})$.

\begin{tabular}{cccc}
\hline Col.1 & Col.2 & Col.3 & Col.4 \\
\hline 13 & 11.61 & 10.93 & 10.48 \\
13 & 11.61 & 10.93 & 10.48 \\
13 & 11.61 & 10.93 & 10.48 \\
\hline
\end{tabular}

Table 7. MRPC of Case 2 (Mvar).

\begin{tabular}{cccc}
\hline Col.1 & Col.2 & Col.3 & Col.4 \\
\hline 0.44 & 1.43 & 1.62 & 1.71 \\
0.44 & 1.43 & 1.62 & 1.71 \\
0.44 & 1.43 & 1.62 & 1.71 \\
\hline
\end{tabular}

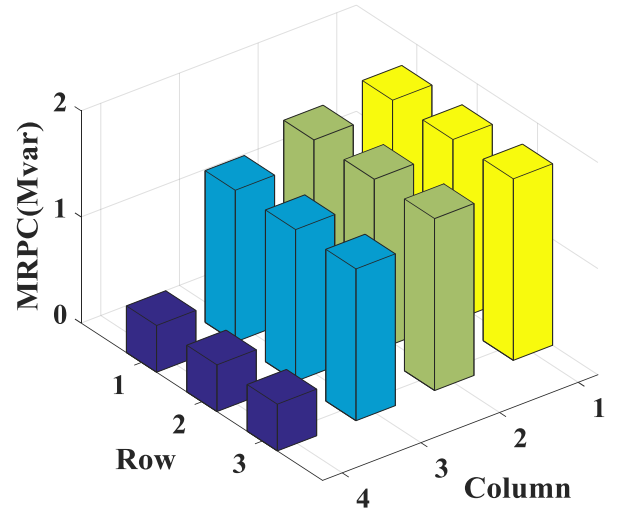

Figure 10. MRPC of the all PMSGs in case 2. 
Table 8. Droop gain of case 2 .

\begin{tabular}{cccc}
\hline Col.1 & Col.2 & Col.3 & Col.4 \\
\hline 11.85 & 38.5 & 43.62 & 46.04 \\
11.85 & 38.5 & 43.62 & 46.04 \\
11.85 & 38.5 & 43.62 & 46.04 \\
\hline
\end{tabular}

Figure 11 shows the result of Case 2, which is identical to case 1 except for the wind direction. As shown in Figure 11a, the voltage nadirs of three schemes (i.e., no voltage control, FGCS and AGCS) after the disturbance are 0.889 p.u., 0.95 p.u. and 0.9549 p.u., respectively. Apparently, the steady state voltage of the proposed scheme is higher than that of the fixed scheme by 0.0049 p.u. and higher than that of "no voltage control scheme" by 0.0659 p.u. This is because the AGCS releases more reactive power than the FGCS during the disturbance.

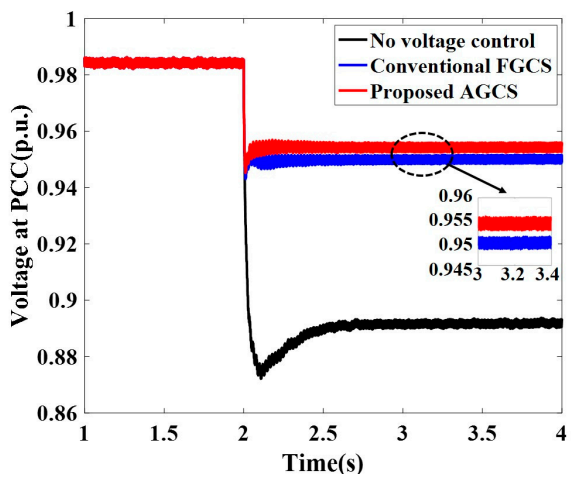

(a)

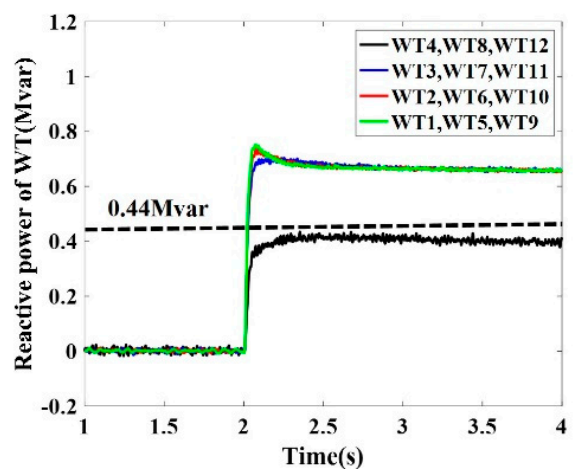

(b)

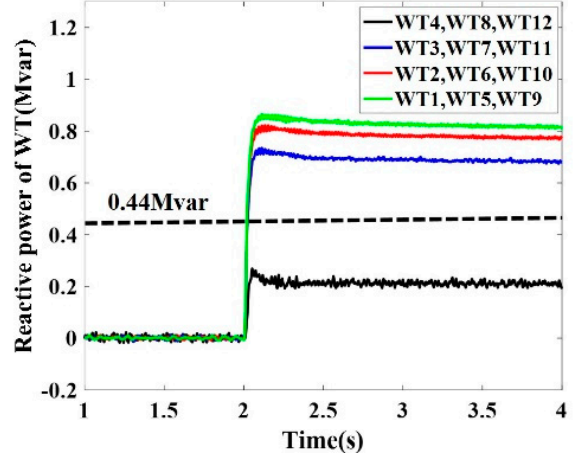

(c)

Figure 11. Results of Case 2. (a) Voltage at PCC, (b) reactive power of PMSGs under the conventional FGCS, (c) reactive power of PMSGs under the proposed AGCS. 
Similarly, as shown in Figure 11b, all PMSGs have the same reactive power generation within the MRPC range for the FGCS, whereas the reactive power generated by fourth column WTs (i.e., WT4, WT8, WT12) is constrained to 0.44 Mvar due to the maximum reactive operating limits.

The comparison of Figures $9 \mathrm{c}$ and $11 \mathrm{c}$ shows that the MRPCs and the droop gains of WTs (i.e., WT1, WT2, WT3) have become larger due to the changes of wind direction. Similarly, the downstream WTs have larger MRPC and the droop gain depends on the spatially different level of MRPC. The adaptive gains and the reactive power generation of WTs (i.e., WT4, WT8, WT12) are smaller than that of the FGCS to avoid maximum reactive output limit, whereas the adaptive gains and the reactive power generation of other WTs are larger than that of the FGCS to generate a certain amount of reactive power.

The results of the above two cases clearly indicate that the AGCS can improve the voltage nadir and ensure the stable operation by setting the spatially dependent gains to PMSGs, especially under high wind speed conditions.

\subsection{Variable Wind Speed}

As mentioned above, the performance of voltage control is critically depending on the MRPC, which can be decreased because of an increase in wind speed. Thus, this section investigates the test results for three cases in which the wind speed increases to a high wind speed and with random fluctuations, respectively.

\subsubsection{Case 3: Increasing Wind Speed from 13 to $14 \mathrm{~m} / \mathrm{s}$ and Wind Direction of $45^{\circ}$}

Figure 12 shows the results in which the wind speed starts increasing from 13 to $14 \mathrm{~m} / \mathrm{s}$ at $4 \mathrm{~s}$, and wind direction is $45^{\circ}$. However, only the fourth column and first row WTs (i.e., WT1, WT2, WT3, WT4, WT8, WT12) experience the wind speed reduction during the simulation time due to the travel time of the wake wind, as shown in Table 9 and Figure 12a.

Table 9. Wind speed of Case $3(\mathrm{~m} / \mathrm{s})$.

\begin{tabular}{cccc}
\hline Col.1 & Col.2 & Col.3 & Col.4 \\
\hline $13 \rightarrow 14$ & $13 \rightarrow 14$ & $13 \rightarrow 14$ & $13 \rightarrow 14$ \\
$13 \rightarrow 14$ & 11.68 & 11.68 & 11.68 \\
$13 \rightarrow 14$ & 11.68 & 10.97 & 10.97 \\
\hline
\end{tabular}

Because of the wind speed change from 13 to $14 \mathrm{~m} / \mathrm{s}$, the WPP has less MRPC than in Case 1, and the droop gains of PMSGs are adaptively changed by using (12), as shown in Tables 10 and 11.

Table 10. MRPC of Case 3 (Mvar).

\begin{tabular}{cccc}
\hline Col.1 & Col.2 & Col.3 & Col.4 \\
\hline $0.44 \rightarrow 0$ & $0.44 \rightarrow 0$ & $0.44 \rightarrow 0$ & $0.44 \rightarrow 0$ \\
$0.44 \rightarrow 0$ & 1.41 & 1.41 & 1.41 \\
$0.44 \rightarrow 0$ & 1.41 & 1.62 & 1.62 \\
\hline
\end{tabular}

Table 11. Droop gain of Case 3.

\begin{tabular}{cccc}
\hline Col.1 & Col.2 & Col.3 & Col.4 \\
\hline $16.04 \rightarrow 0$ & $16.04 \rightarrow 0$ & $16.04 \rightarrow 0$ & $16.04 \rightarrow 0$ \\
$16.04 \rightarrow 0$ & $51.40 \rightarrow 66.68$ & $51.40 \rightarrow 66.68$ & $51.40 \rightarrow 66.68$ \\
$16.04 \rightarrow 0$ & $51.40 \rightarrow 66.68$ & $59.07 \rightarrow 76.42$ & $59.07 \rightarrow 76.42$ \\
\hline
\end{tabular}




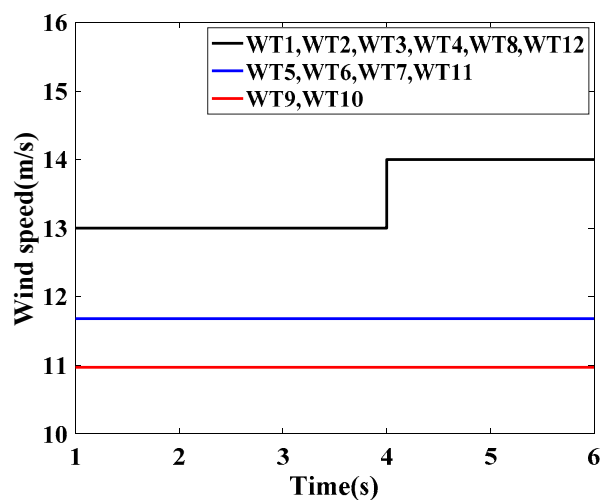

(a)

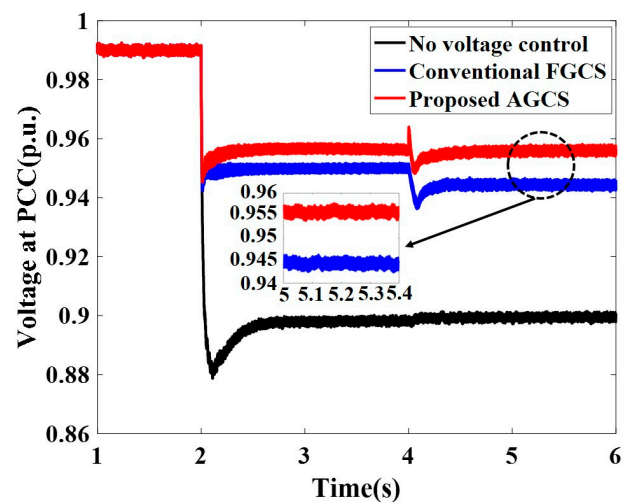

(b)

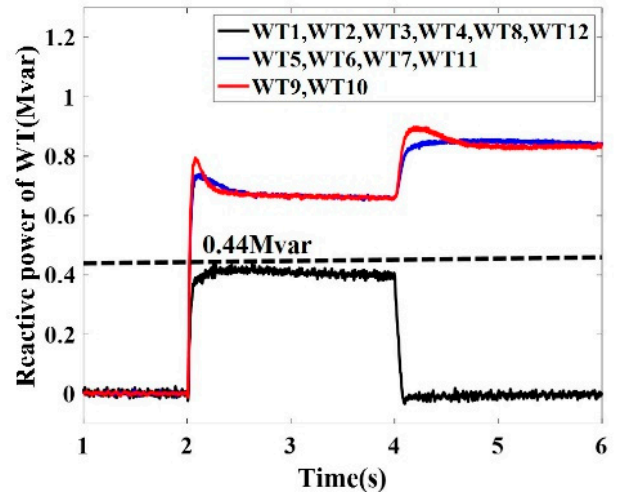

(c)

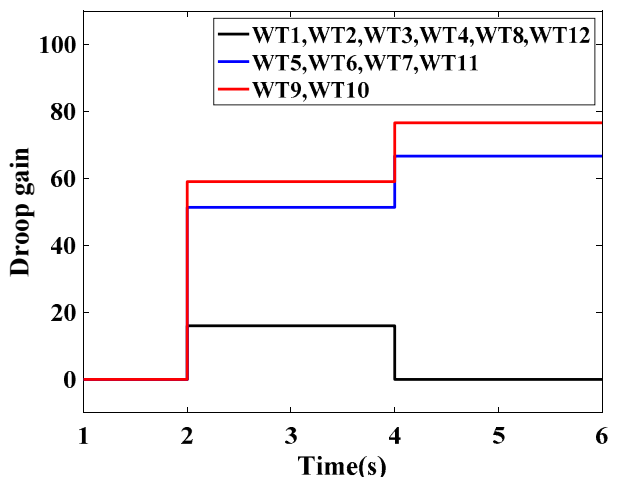

(d)

Figure 12. Cont. 


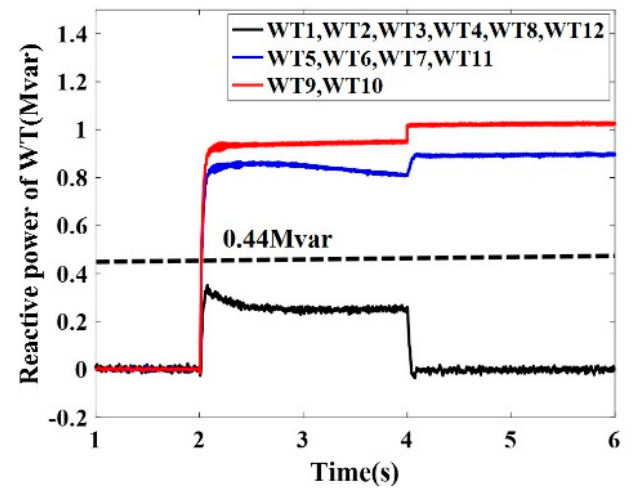

(e)

Figure 12. Results of Case 3. (a) Wind speed, (b) voltage at PCC, (c) reactive power of PMSGs under the conventional FGCS, (d) adaptive gain of PMSGs, (e) reactive power of PMSGs under the proposed AGCS.

As shown in Figure 12b, the voltage nadir of FGCS and AGCS after the disturbance of wind speed changes are 0.9448 p.u. and 0.957 p.u., respectively. Apparently, the steady state voltage of the proposed scheme is higher than that of the fixed scheme by 0.0122 p.u. Because the input wind speed of the upstream WTs (i.e., fourth column and first row WTs) exceeds the rated operation speed, the WTs output the maximum rated active power, thus there is no reactive power output capability for the converters of these PMSGs.

For the FGCS, the reactive power generated by the fourth column and first row WTs (i.e., WT1, WT2, WT3, WT4, WT8, WT12) is constrained to 0 Mvar due to the limit of reactive power. The unfavorable result is increasing wear and tear to the converters. The reactive power generated by other WTs is increased due to the larger voltage deviation at PCC caused by the disturbance of increased wind speed, as shown in Figure 12c. However, for the AGCS, the reactive power generated by the fourth column and first row WTs (i.e., WT1, WT2, WT3, WT4, WT8, WT12) becomes 0 Mvar because of the adaptive change in droop gain (changed to 0 ) rather than the reactive power limitation. The reactive power generated by other WTs is increased, this is not only because the voltage deviation at PCC becomes larger, but also the droop gains of these PMSGs are increased adaptively by using (12), as shown in Figure 12d,e, respectively.

\subsubsection{Case 4: Increasing Wind Speed from 13 to $14 \mathrm{~m} / \mathrm{s}$ and Wind Direction of $0^{\circ}$}

Figure 13 shows the results of Case 4, which is identical to Case 3 except for the wind direction. In this Case, the wind direction is changed to $0^{\circ}$, and only the fourth column WTs (i.e., WT4, WT8, WT12) experience the wind speed reduction during the simulation time due to the travel time of the wake wind, as shown in Table 12 and Figure 13a. Similarly, the WPP has less MRPC than in Case 2, and the droop gains of PMSGs are adaptively changed by using (12), as shown in Tables 13 and 14.

The voltage nadir of FGCS and AGCS after the disturbance of wind speed changes are 0.9456 p.u. and 0.9549 p.u., respectively. The steady state voltage of the proposed scheme is higher than that of the fixed scheme by 0.0093 p.u.. The reason for the change trend of the reactive power of FGCS, the reactive power and the droop gains of AGCS are the same as described in Case 3. However, compared with the three WT groups in Case 3, the wind speed, MRPC and droop gains are divided into four groups in this case, and the biggest difference is that the wind speed, MRPC and droop gains of the first row WTs of WPP has undergone major changes due to the wind direction change from $45^{\circ}$ to $0^{\circ}$. 


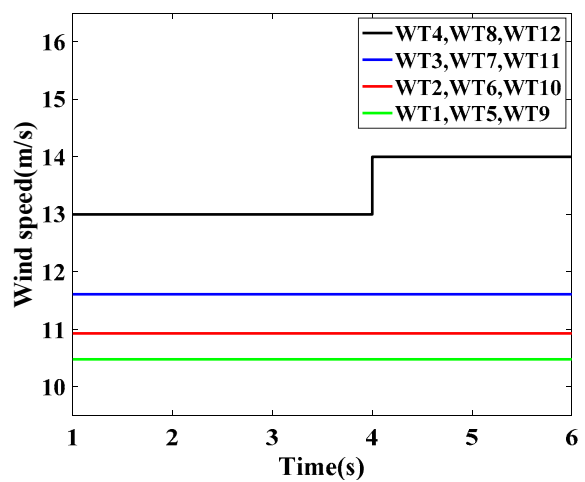

(a)

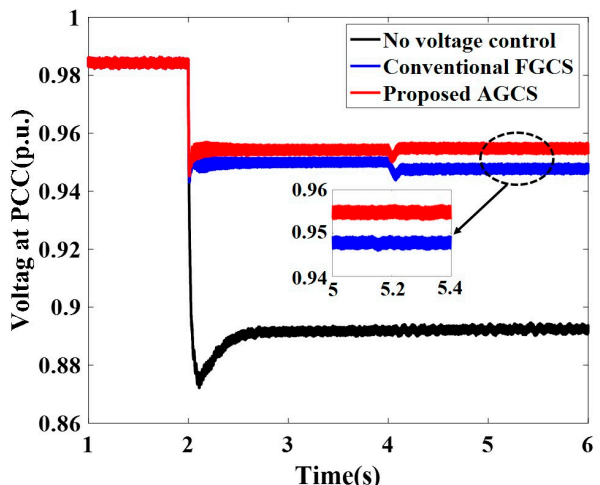

(b)

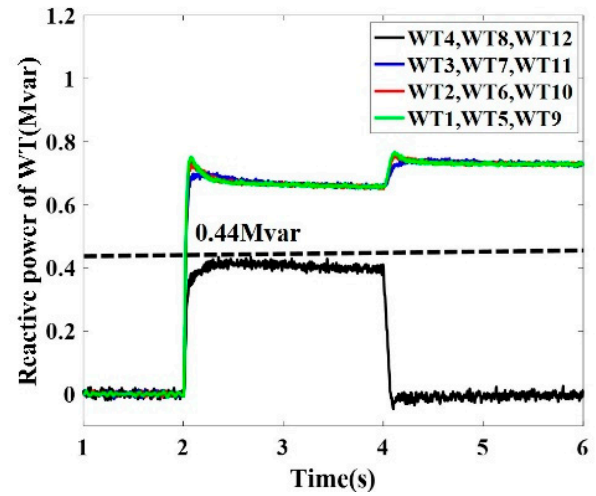

(c)

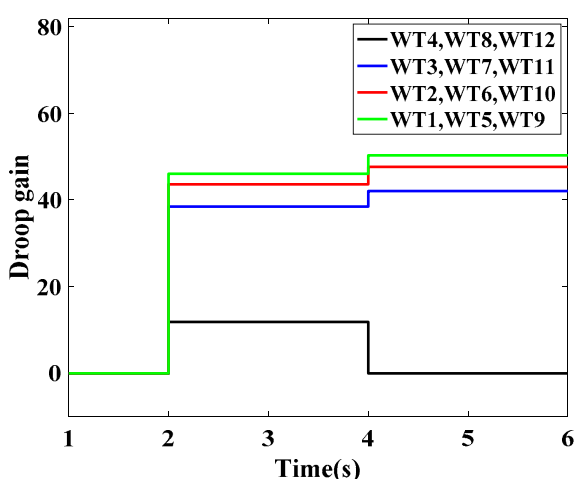

(d)

Figure 13. Cont. 


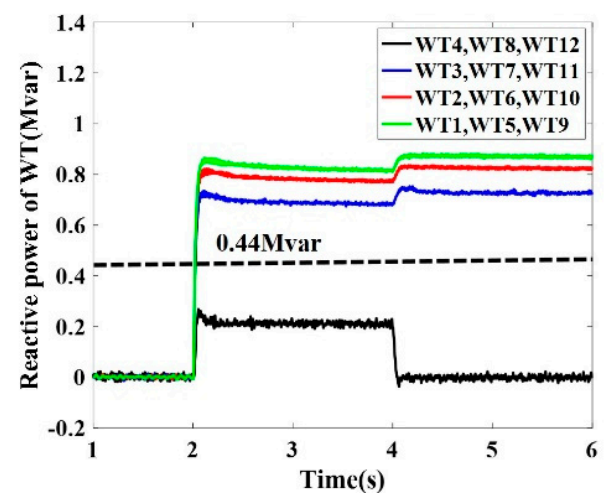

(e)

Figure 13. Results of Case 4. (a) Wind speed, (b) voltage at PCC, (c) reactive power of PMSGs under the conventional FGCS, (d) adaptive gain of PMSGs, (e) reactive power of PMSGs under the proposed AGCS.

\subsubsection{Case 5: Real Pattern of Wind Speed, and Wind Direction of $0^{\circ}$}

In reality, the wind speed changes all the time. In this case, a real wind speed pattern that have specific statistical features and wind direction of $0^{\circ}$ is employed [26], as shown in Figure 14a.

Table 12. Wind speed of Case $3(\mathrm{~m} / \mathrm{s})$.

\begin{tabular}{cccc}
\hline Col.1 & Col.2 & Col.3 & Col.4 \\
\hline $13 \rightarrow 14$ & 11.61 & 10.93 & 10.48 \\
$13 \rightarrow 14$ & 11.61 & 10.93 & 10.48 \\
$13 \rightarrow 14$ & 11.61 & 10.93 & 10.48 \\
\hline
\end{tabular}

Table 13. MRPC of Case 3 (Mvar).

\begin{tabular}{cccc}
\hline Col.1 & Col.2 & Col.3 & Col.4 \\
\hline $0.44 \rightarrow 0$ & 1.43 & 1.62 & 1.71 \\
$0.44 \rightarrow 0$ & 1.43 & 1.62 & 1.71 \\
$0.44 \rightarrow 0$ & 1.43 & 1.62 & 1.71 \\
\hline
\end{tabular}

Table 14. Droop gain of Case 3.

\begin{tabular}{cccc}
\hline Col.1 & Col.2 & Col.3 & Col.4 \\
\hline $11.85 \rightarrow 0$ & $38.5 \rightarrow 42.06$ & $43.62 \rightarrow 47.65$ & $46.04 \rightarrow 50.29$ \\
$11.85 \rightarrow 0$ & $38.5 \rightarrow 42.06$ & $43.62 \rightarrow 47.65$ & $46.04 \rightarrow 50.29$ \\
$11.85 \rightarrow 0$ & $38.5 \rightarrow 42.06$ & $43.62 \rightarrow 47.65$ & $46.04 \rightarrow 50.29$ \\
\hline
\end{tabular}

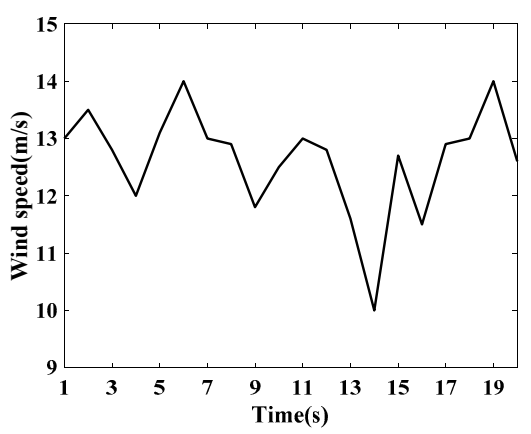

(a)

Figure 14. Cont. 


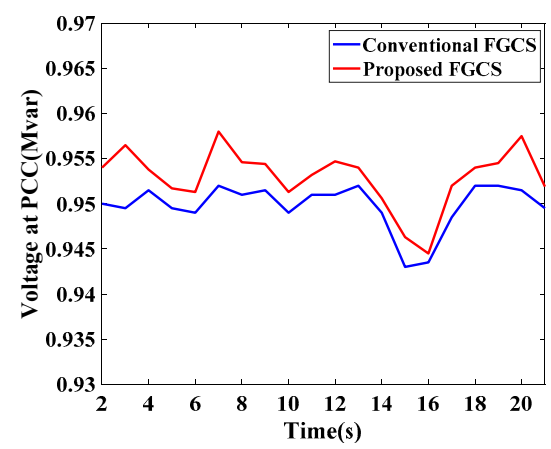

(b)

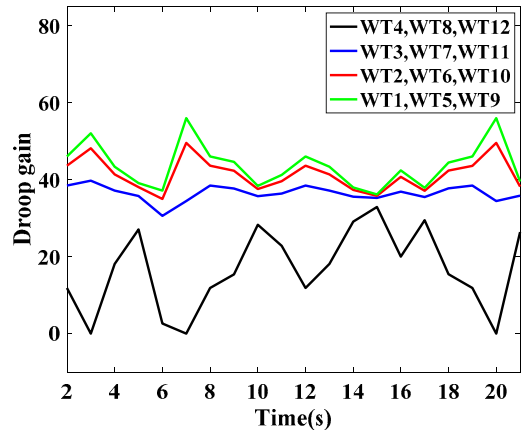

(c)

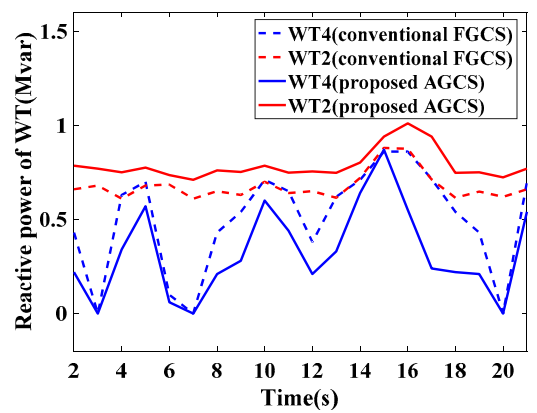

(d)

Figure 14. Results of Case 5. (a) Real pattern of wind speed, (b) voltage at PCC, (c) adaptive gain of PMSGs, (d) reactive power of PMSGs under the proposed AGCS.

The voltage nadir of FGCS and AGCS after the disturbance are compared as shown in Figure 14b. It can be seen that the AGCS exhibits better performance in improving the voltage nadir at PCC.

It should be emphasized that for conventional FGCS the reactive power generated by the fourth column WTs (i.e., WT4, WT8, WT12) will frequently exceed the limit. This results in not only limiting the support for the voltage at the PCC point, but also causing the wear outs to the PMSG converters. However, for the proposed AGCS, $v_{i}$ and $A G_{i}\left(v_{i}\right)$ are spatially and temporally dependent variables, thus the droop gains of PMSGs are adaptively changed by using (12). Consequently, the downstream WTs have higher droop gain, and the result is that not only the reactive power generated by the PMSGs does not exceed the MRPC to avoid the wear outs to the WT converters, but also provides more effective voltage support for the PCC point, as shown in Figure 14c,d. Specifically, the AGCS shows better performance at high wind speed.

The results of Case 3, Case 4 and Case 5 demonstrate that the AGCS ensures stable operation of all PMSGs to avoid the wear outs to the WT converters while improving the voltage nadir by setting the spatially and temporally dependent gains to PMSGs at the disturbance instant when wind speed significantly fluctuates. 


\section{Conclusions}

This paper proposes a control scheme with adaptive gains for a PMSG-based WPP. The droop gains of PMSGs are adjusted adaptively based on the MRPC, which is closely related to input wind speed. To do this, a function that relates the droop gain to the wind speed is used. The different gains are set to be proportional to the spatially and temporally different levels of MRPC. To improve the voltage-supporting capacity, the droop gains of downstream WTs are adjusted to a larger value due to the larger MRPC; then, to ensure stable operation of all PMSGs, the droop gains of upstream WTs are adjusted to a smaller value due to the smaller MRPC.

Various simulation results demonstrate that the proposed scheme has better performance than the conventional FGCS in terms of improving the voltage nadir at PCC and ensuring stable operation of all PMSGs in WPP under load disturbance and various wind conditions (i.e., different wind direction, wind speed increase and wind speed fluctuation). The advantage of the proposed scheme is that it not only improves the voltage nadir by making full use of the WPP reactive capacity but also presents PMSGs at a high wind speed from exceeding the maximum reactive capacity. Moreover, the proposed scheme has application potential in a power system with high wind power penetration.

Author Contributions: J.D. and Y.T. conceived and designed the study; J.D. analyzed the data, performed the experiments and wrote the paper; J.Y. provided some actual wind power parameters and checked the results of this work. All the authors read and approved the final manuscript.

Funding: This research and the APC were funded by [the Technology Project of the State Grid Electric Power Company (Study on power generation control system and operation control strategy of super-high-ratio new energy grid)".

Conflicts of Interest: The authors declare no conflict of interest.

\section{References}

1. Global Wind Energy Outlook. 2017. Available online: http://www.gwec.net (accessed on 20 December 2018).

2. Huenteler, J.; Niebuhr, C.; Schmidt, T.S. The effect of local and global learning on the cost of renewable energy in developing countries. J. Clean. Prod. 2016, 128, 6-21. [CrossRef]

3. Lind, P.G.; Vera-Tudela, L.; Wächter, M.; Kühn, M.; Peinke, J. Normal behaviour models for wind turbine vibrations: Comparison of neural networks and a stochastic approach. Energies 2017, 10, 1944. [CrossRef]

4. Muljadi, E.; Butterfield, C.P.; Parsons, B.; Ellis, A. Effect of variable speed wind turbine generator on stability of a weak grid. IEEE Trans. Energy Convers. 2007, 22, 29-36. [CrossRef]

5. Tsili, M.; Papathanassiou, S. A review of grid code technical requirements for wind farms. IET Renew. Power Gener. 2009, 3, 308-332. [CrossRef]

6. Yassin, H.M.; Hanafy, H.H.; Hallouda, M.M. Enhancement low-voltage ride through capability of permanent magnet synchronous generator-based wind turbines using interval type-2 fuzzy control. IET Renew. Power Gener. 2016, 10, 339-348. [CrossRef]

7. Tao, R.; Li, F.; Chen, W.; Fan, Y.; Liang, C.; Li, Y. Research on the protection coordination of permanent magnet synchronous generator based wind farms with low voltage ride through capability. Prot. Control Modern Power Syst. 2017, 2, 28. [CrossRef]

8. Guo, Q.; Sun, H.; Wang, B.; Zhang, B.; Wu, W.; Lei, T. Hierarchical automatic voltage control for integration of large-scale wind power: Design and implementation. Electr. Power Syst. Res. 2015, 120, 234-241. [CrossRef]

9. Khezri, R.; Bevrani, H. Voltage performance enhancement of DFIG-based wind farms integrated in large-scale power systems: Coordinated AVR and PSS. Int. J. Electr. Power Energy Syst. 2015, 73, 400-410. [CrossRef]

10. Qin, N.; Bak, C.L.; Abildgaard, H.; Chen, Z. Multi-stage optimization-based automatic voltage control systems considering wind power forecasting errors. IEEE Trans. Power Syst. 2016, 32, 1073-1088. [CrossRef]

11. Lund, T.; Sørensen, P.; Eek, J. Reactive power capability of a wind turbine with doubly fed induction generator. Wind Energy 2010, 10, 379-394. [CrossRef]

12. Ko, H.S.; Yoon, G.G.; Hong, W.P. Active use of DFIG-based variable-speed wind-turbine for voltage regulation at a remote location. IEEE Trans. Power Syst. 2007, 22, 1916-1925. [CrossRef] 
13. Niu, T.; Guo, Q.; Jin, H.; Sun, H.; Zhang, B.; Liu, H. Dynamic reactive power optimal allocation to decrease wind power curtailment in a large-scale wind power integration area. IET Renew. Power Gener. 2017, 11, 1667-1678. [CrossRef]

14. Zuo, W.; Wang, X.; Kang, S. Numerical simulations on the wake effect of H-type vertical axis wind turbines. Energy 2016, 106, 691-700. [CrossRef]

15. Kuenzel, S.; Kunjumuhammed, L.P.; Pal, B.C.; Erlich, I. Impact of Wakes on Wind Farm Inertial Response. IEEE Trans. Sustain. Energy 2013, 5, 237-245. [CrossRef]

16. Duckworth, A.; Barthelmie, R.J. Investigation and Validation of Wind Turbine Wake Models. Wind Eng. 2008, 32, 459-475. [CrossRef]

17. González-Longatt, F.; Wall, P.; Terzija, V. Wake effect in wind farm performance: Steady-state and dynamic behavior. Renew. Energy 2014, 39, 329-338. [CrossRef]

18. Kim, H.W.; Kim, S.S.; Ko, H.S. Modeling and control of PMSG-based variable-speed wind turbine. Electr. Power Syst. Res. 2010, 80, 46-52. [CrossRef]

19. Senjyu, T.; Sakamoto, R.; Urasaki, N.; Funabashi, T.; Fujita, H.; Sekina, H. Output power leveling of wind turbine Generator for all operating regions by pitch angle control. IEEE Trans. Energy Convers. 2010, 162, 17-24. [CrossRef]

20. Cheng, Z.; Lai, W.; Yan, G. Low Voltage ride-through scheme of the PMSG wind power system based on coordinated instantaneous active power control. Energies 2017, 10, 995. [CrossRef]

21. Zhao, Y.; Chai, J.; Sun, X. Relative Voltage Control of the Wind Farms Based on the Local Reactive Power Regulation. Energies 2017, 10, 281. [CrossRef]

22. Lee, J.; Jang, G.; Muljadi, E.; Blaabjerg, F.; Chen, Z.; Cheol Kang, Y. Stable Short-Term Frequency Support Using Adaptive Gains for a DFIG-Based Wind Power Plant. IEEE Trans. Energy Convers. 2016, 31, 1-12. [CrossRef]

23. Kundera, P.; Balu, N.J.; Lauby, M.G. Power System Stability and Control; McGraw-Hill: New York, NY, USA, 1994.

24. IEEE Std. IEEE Recommended Practice for Excitation System Models for Power System Stability Studies; IEEE: Piscataway, NJ, USA, 2016.

25. Kim, S.K.; Kim, E.S. PSCAD/EMTDC-based modelling and analysis of a gearless variable speed wind turbine. IEEE Trans. Energy Convers. 2007, 22, 421-430. [CrossRef]

26. Database of Wind Characteristics Located at DTU. 2017. Available online: http://www.winddata.com (accessed on 13 December 2018). 